\title{
DEGRADAÇÃO DE CONTAMINANTES PRESENTES EM EFLUENTE DA PRODUÇÃO DE QUEIJOS POR PROCESSOS AVANÇADOS USANDO FERRO METÁLICO
}

\author{
P. R. A. MENDES ${ }^{1}$, L. F. F. FARIA ${ }^{1}$ \\ ${ }^{1}$ Escola de Engenharia de Lorena/USP, Departamento de Engenharia Química \\ E-mail para paulo_ricardo_am@yahoo.com.br
}

\begin{abstract}
RESUMO - O uso de ferro metálico $\left(\mathrm{Fe}^{0}\right)$ em substituição ao ferro solúvel (íons ferrosos e férricos) para sistema foto-Fenton vem tendo aplicações crescentes devido à melhoria da eficiência de degradação de muitos compostos recalcitrantes, atuando como forte agente redutor. Outras vantagens como menor quantidade residual ao final do processo, baixo custo, abundância na crosta terrestre e baixa toxicidade são citadas na literatura. Assim, este trabalho propõe a avaliação e otimização das concentrações de peróxido de hidrogênio $\left(\mathrm{H}_{2} \mathrm{O}_{2}\right)$ e de $\mathrm{Fe}^{0}$, por meio de planejamento experimental do tipo fatorial, sobre as variáveis respostas carbono orgânico total (COT) e demanda química de oxigênio (DQO). Como fontes de $\mathrm{Fe}^{0}$ foram testadas o ferro granulado $(5 \mu \mathrm{m})$ e lã de aço comercial (fios com diâmetro médio de $0,03 \mathrm{~mm}$ ). Os melhores resultados foram obtidos a partir do uso da lã de aço confinada. Na condição ótima $\left(580 \mathrm{mM}\right.$ para $\mathrm{H}_{2} \mathrm{O}_{2}$ e $26,6 \mathrm{mM}$ para $\mathrm{Fe}^{0}$ ) foram obtidas reduções de 74,9\% em COT e 41,0\% em DQO.
\end{abstract}

\section{INTRODUÇÃO}

Os efluentes gerados pelas indústrias produtoras de queijo poluem devido a sua elevada quantidade de matéria orgânica (DQO: 800-102.000 mg L ${ }^{-1}$; DBO: 600-60.000 mg L-1), devendo ser submetido a tratamentos específicos antes do seu despejo (Prazeres et al., 2012; Baldasso et al., 2011). O soro de queijo é obtido a partir da produção de queijo ou da manufatura da caseína presente no leite (Prazeres et al., 2012; Cuartas-Uribe et al., 2009). Considerando a produção brasileira de queijo em 2013 e a relação média de 10 litros de soro gerados por cada quilo de queijo produzido, neste ano, foram gerados cerca de 7 bilhões de litros de soro (Mapa, 2014), representando uma quantidade bastante significativa se não for devidamente tratada.

Uma das tecnologias para o tratamento de efluentes complexos como o de laticínios são os Processos Oxidativos Avançados (POAs) utilizados para a degradação de poluentes, principalmente os recalcitrantes. Estes processos exploram as ações dos radicais hidroxila, espécies oxidantes altamente reativas de vida curta com potencial de redução de 2,8 V. Nos POAs a geração destes radicais é o passo fundamental para a eficiência do processo. Quanto mais eficientemente estes radicais forem gerados maior será o poder oxidativo (Galvez; Rodriguez, 2003; Oppenländer, 2003). No entanto, os POAs apresentam limitações para 
decomposição de compostos que apresentam centros deficientes de elétrons, pois a reação do radical hidroxila é bem mais lenta. Assim, os processos com aplicação de radiação UV ou visível exibem deficiências para a degradação de substratos com grande quantidade de sólidos suspensos (Pereira et al, 2005; Souza, 2006).

Uma possível aplicação do ferro metálico $\left(\mathrm{Fe}^{0}\right)$ como catalisador vem sendo estudada por apresentar inúmeras vantagens como, por exemplo, abundância do metal na crosta terrestre, baixa toxicidade, baixo custo, sua efetividade como agente redutor e reutilização do ferro, não havendo necessidade de remoção que se fazem necessários nos processos Fenton/foto-Fenton (Epolito et al., 2008). O ferro metálico apresenta um potencial padrão de redução para o $\mathrm{Fe}^{2+}$ de $-0,44 \mathrm{~V}$, sendo relativamente forte quando comparado a uma série de substâncias orgânicas. Pode-se afirmar que na redução de ferro metálico há a geração constante de íons $\mathrm{Fe}^{2+}$, que podem ser utilizados em reações do tipo Fenton (Pereira et al, 2005; Souza, 2006). Assim, quando o ferro metálico é usado como catalisador em reações Fenton e foto-Fenton o processo é denominado de Fenton avançado, pois substitui os sais de ferro evitando a adição desnecessária de cargas aniônicas no efluente em estudo e, ainda, promover a rápida redução dos íons $\mathrm{Fe}^{+3}$ a $\mathrm{Fe}^{+2}$ na superfície do $\mathrm{Fe}^{0}$, conforme a Equação 1 (Bremner et al., 2006; Kušić et al., 2007; Zhang et al., 2009).

$$
2 \mathrm{Fe}^{3+}+\mathrm{Fe}^{0} \rightarrow 3 \mathrm{Fe}^{2+}
$$

Os elétrons produzidos a partir da oxidação do $\mathrm{Fe}^{0}$ a $\mathrm{Fe}^{2+}$ também podem ser capturados por espécies oxidantes presentes no meio reacional pois, na presença do $\mathrm{H}_{2} \mathrm{O}_{2}$, ocorre a oxidação do $\mathrm{Fe}^{0}$ para $\mathrm{Fe}^{2+}$ conforme indicado na Equação (2) (Bergendahl, Thies, 2004).

$$
\mathrm{Fe}^{0}+\mathrm{H}_{2} \mathrm{O}_{2} \rightarrow \mathrm{Fe}^{2+}+2 \mathrm{HO}^{-}
$$

Desta maneira, a associação de processos redutivos e oxidativos primeiramente com a passagem do $\mathrm{Fe}^{0}$ a Fe ${ }^{2+/ 3+}$ através da oxidação do $\mathrm{Fe}^{0}$ promovida pela água e $\mathrm{H}_{2} \mathrm{O}_{2}$ (processo redutivo), o ferro oxidado (íons $\mathrm{Fe}^{2+} / \mathrm{Fe}^{3+}$ ) decorrente desta etapa é sequencialmente aproveitado como fonte de íons ferro para os processos Fenton e foto-Fenton, podendo alcançar uma sinergia e realizar a degradação e/ou mineralização a níveis significativos de uma série de recalcitrantes (Pereira; Freire, 2005; Souza; Peralta-Zamora, 2005).

Em vista disso, objetivando minimizar os impactos do descarte do efluente da produção de queijo e assim atender as exigências ambientais, o presente trabalho propõe uma alternativa para o tratamento destes resíduos, visando à redução de contaminantes orgânicos e aumento da biodegradabilidade por POAs, especificamente o Processo Foto-Fenton avançado.

\section{MATERIAIS E MÉTODOS}

O efluente foi gentilmente cedido pela empresa Danubio (Dan Vigor Indústria e Comércio de Laticínios Ltda.), sediada na cidade de Cruzeiro/SP, Brasil. Neste material foram adicionados $0,10 \%$ de biocida IPEL BP-15 para evitar a contaminação e conservado em câmara fria a $4{ }^{\circ} \mathrm{C}$, para manter suas características físico-químicas durante os tratamentos. 
$\mathrm{Na}$ caracterização da amostra, os parâmetros $\mathrm{pH}$, turbidez, condutividade, COT, DQO, DBO, sólidos totais e sólidos suspensos foram determinados conforme Standard Methods of Examination of Water and Wastewater $20^{\text {th }}$ ed. A lactose foi determinada por HPLC de fase reversa. Os principais reagentes utilizados foram $\mathrm{H}_{2} \mathrm{O}_{2}$ P.A. $30 \%$, lã de aço comercial Bombril $^{\circledR}$, soluções de $\mathrm{H}_{2} \mathrm{SO}_{4} 5 \mathrm{~N}$ e NaOH 6,5N para o ajuste do $\mathrm{pH}$.

Os experimentos foram realizados a temperatura ambiente $\left(25^{\circ} \mathrm{C}\right)$ em um fotorreator encamisado de 3 litros, com poço anular em quartzo como sede da fonte de radiação UV e banho ultratermostatizado. O poço anular de quartzo e o fotorreator são encamisados com circulação água a fim de manter a temperatura ao longo da reação. A homogeneização do sistema foi garantida pela recirculação do soro por meio de bomba de recirculação. A lã de aço, como fonte de ferro metálico $\left(\mathrm{Fe}^{0}\right)$, foi confinada em um recipiente plástico de $50 \mathrm{~mL}$, com pequenas perfurações na base, conectada a linha de recirculação do meio. A radiação UV foi gerada a partir de lâmpada de $250 \mathrm{~W}$ de alta pressão de vapor de mercúrio. A determinação da condição ótima iniciou-se a partir de planejamento exploratório fatorial completo $2^{2}$ com triplicata no ponto central, totalizando 7 experimentos (tabela 1). Foram avaliadas a influência das variáveis controle concentrações de $\mathrm{H}_{2} \mathrm{O}_{2}$ e de $\mathrm{Fe}^{0}$ sobre as variáveis resposta de reduções de COT e de DQO. $\mathrm{O} \mathrm{H}_{2} \mathrm{O}_{2}$ foi adicionado gradativamente em um intervalo de 30 minutos. Foram retiradas amostras a cada 15 minutos, a fim de monitorar o esgotamento total do $\mathrm{H}_{2} \mathrm{O}_{2}$ em um tempo de reação de 2 horas.

Tabela 1 - Variáveis de controle e respectivos níveis do planejamento fatorial $2^{2}$.

\begin{tabular}{cccc}
\hline \multirow{2}{*}{ Variáveis de Controle } & \multicolumn{3}{c}{ Níveis } \\
\cline { 2 - 4 } & Baixo (-1) & Central (0) & Alto (+1) \\
\hline $\mathrm{H}_{2} \mathrm{O}_{2}(\mathrm{mM})$ & 353 & 530 & 707 \\
$\mathrm{Fe}^{0}(\mathrm{mM})$ & 8,95 & 17,9 & 26,9 \\
\hline
\end{tabular}

\section{RESULTADOS E DISCUSSÃO}

Após coleta do efluente gerado, amostras foram caracterizadas e os resultados são mostrados na Tabela 2. $\mathrm{O}$ remanescente foi encaminhado à conservação. $\mathrm{O}$ efluente bruto apresentou valores elevados de DQO e COT, indicando a presença de carboidratos como matéria orgânica dissolvida, principalmente, devido à presença de lactose. $O$ fato deste efluente não apresentar turbidez, a quantidade de sólidos suspensos determinada não foi significativa comparada com a dos sólidos totais. A razão DBO/DQO obtida para este efluente é de 0,35 configurando que este efluente ocasionaria dificuldades para degradação em sistemas biológicos. Em seguida, o efluente caracterizado foi submetido aos testes de oxidação avançada de acordo com o planejamento proposto e as condições experimentais e os resultados obtidos são mostrados na Tabela 3 .

Para avaliar o nível de influência das variáveis controle, os resultados obtidos foram tratados estatisticamente a um nível de significância de $95 \%$ com auxílio do software Minitab $^{\circledR}$. Ao analisar os resultados, foi possível observar que todas as variáveis controle 
apresentaram significância estatística para ambas as variáveis resposta, com exceção da redução de $\mathrm{COT}$, em que somente a concentração de $\mathrm{Fe}^{0}$ foi significativa (Figura 1). Pela análise de variância (ANOVA), os modelos gerados para as reduções de COT e DQO indicaram a presença de curvatura na região estudada, podendo ser utilizado modelos quadráticos para ajuste dos resultados (Tabelas 4 e 5).

Tabela 2 - Caracterização do efluente bruto.

\begin{tabular}{cc}
\hline Parâmetros & Valores \\
\hline $\mathrm{pH}$ & 6,58 \\
Condutividade $\left(\mathrm{mS} \mathrm{cm}^{-1}\right)$ & 4,76 \\
DQO $\left(\mathrm{mg} \mathrm{L}^{-1}\right)$ & 2310 \\
COT $\left(\mathrm{mg} \mathrm{L}^{-1}\right)$ & 534 \\
DBO $\left(\mathrm{mg} \mathrm{L}^{-1}\right)$ & 804 \\
Turbidez $\left(\mathrm{NTU}^{-1}\right)$ & 0,05 \\
Sólidos totais $\left(\mathrm{mg} \mathrm{L}^{-1}\right)$ & 4835 \\
Sólidos dissolvidos $\left(\mathrm{mg} \mathrm{L}^{-1}\right)$ & 4835 \\
Lactose $\left(\mathrm{g} \mathrm{L}^{-1}\right)$ & 1,21 \\
\hline
\end{tabular}

Tabela 3 - Matriz experimental do planejamento fatorial $2^{2}$ e respectivos valores de redução de COT e DQO.

\begin{tabular}{ccccc}
\hline Exp. & $\begin{array}{c}\mathbf{H}_{\mathbf{2}} \mathbf{O}_{\mathbf{2}} \\
(\mathbf{m M})\end{array}$ & $\begin{array}{c}\mathbf{F e}^{\mathbf{0}} \\
(\mathbf{m M})\end{array}$ & $\begin{array}{c}\text { Redução } \\
\mathbf{C O T}(\boldsymbol{\%})\end{array}$ & $\begin{array}{c}\text { Redução } \\
\text { DQO }(\%)\end{array}$ \\
\hline 1 & 353 & 8,95 & 69,0 & 39,4 \\
2 & 707 & 8,95 & 69,3 & 38,2 \\
3 & 353 & 26,9 & 72,5 & 39,8 \\
4 & 707 & 26,9 & 72,4 & $\mathbf{4 3 , 9}$ \\
5 & 530 & 17,9 & $\mathbf{7 3 , 8}$ & 37,6 \\
6 & 530 & 17,9 & $\mathbf{7 3 , 3}$ & 36,7 \\
7 & 530 & 17,9 & $\mathbf{7 3 , 1}$ & 37,8 \\
\hline
\end{tabular}

Avaliando o consumo de $\mathrm{H}_{2} \mathrm{O}_{2}$ ao longo da reação, foi constatado que todos os experimentos obtiveram taxas de consumo de $\mathrm{H}_{2} \mathrm{O}_{2}$ inferiores a $70 \%$ para tempo de reação de até 120 minutos e que para experimentos de 1 a 4, maiores concentrações de $\mathrm{Fe}^{0}$ favoreceram um maior consumo de $\mathrm{H}_{2} \mathrm{O}_{2}$. Este efeito é favorecido devido a uma concentração maior de $\mathrm{Fe}^{0}$ inicial disponível. Para uma mesma concentração de $\mathrm{Fe}^{0}$, quando há aumento na concentração inicial de $\mathrm{H}_{2} \mathrm{O}_{2}$, o consumo não teve alteração significativa.

(a)

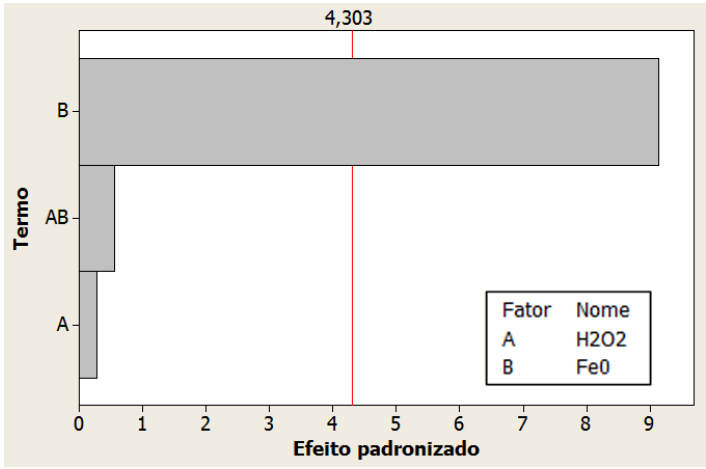

(b)

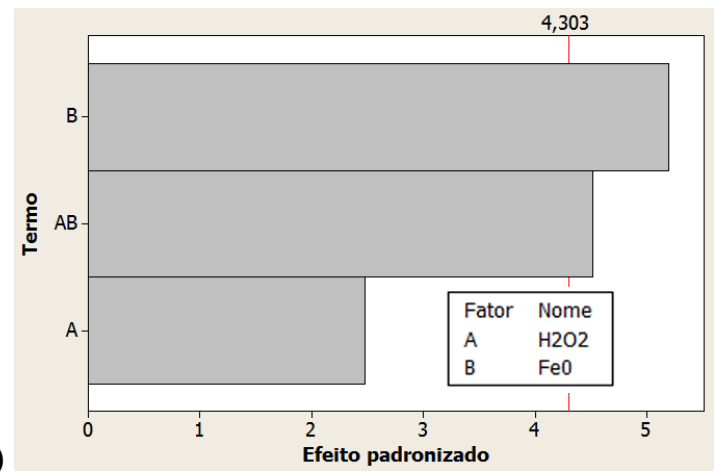

Figura 1 - Diagramas de Pareto para reduções de COT (a) e DQO (b). Planejamento fatorial $2^{2}$. 
Tabela 4 - ANOVA para a redução de COT do planejamento fatorial $2^{2}$.

\begin{tabular}{ccccc}
\hline Fonte de Variação & SQ & Graus de Liberdade & MQ & p - valor \\
\hline Regressão & 10,9000 & 2 & 5,4500 & 0,023 \\
Interação de 2 $^{\mathbf{a}}$ ordem & 0,0400 & 1 & 0,0400 & 0,635 \\
Curvatura & 11,5886 & 1 & 11,5886 & 0,011 \\
Resíduo & 0,2600 & 2 & 0,1300 & \\
Erro Puro & 0,2600 & 2 & 0,1300 & \\
Total & 22,7886 & 6 & & \\
\hline
\end{tabular}

Tabela 5 - ANOVA para a redução de DQO do planejamento fatorial $2^{2}$.

\begin{tabular}{ccccc}
\hline Fonte de Variação & SQ & Graus de Liberdade & MQ & p - valor \\
\hline Regressão & 11,4050 & 2 & 5,7025 & 0,057 \\
Interação de 2 $^{\mathbf{a}}$ ordem & 7,0225 & 1 & 7,0225 & 0,046 \\
Curvatura & 15,0030 & 1 & 15,0030 & 0,022 \\
Resíduo & 0,6867 & 2 & 0,3433 & \\
Erro Puro & 0,6867 & 2 & 0,3433 & \\
Total & 34,1171 & 6 & & \\
\hline
\end{tabular}

Considerando os resultados anteriores com relação os níveis escolhidos da variável concentração de $\mathrm{H}_{2} \mathrm{O}_{2}$ para o fatorial completo, observa-se que estes valores já contemplaram uma região de máximo para a resposta redução de COT. Assim, os mesmos níveis foram mantidos para o Delineamento Composto Central Rotacional (DCCR). No entanto, a concentração de $\mathrm{Fe}^{0}$ era duvidosa no meio reacional, pois para os experimentos 3 e 4 do fatorial completo foi observado que ao dobrar a quantidade de $\mathrm{H}_{2} \mathrm{O}_{2}$ para condição máxima de ferro $(26,9 \mathrm{mM})$ não ocorreu alteração considerável na redução de COT. A partir disso foi possível observar a existência de limitação causada pela cinética de transferência de íons ferrosos do $\mathrm{Fe}^{0}$ (sólido) para o meio reacional. Assim, na tentativa de definir níveis mais precisos para o $\mathrm{Fe}^{0}$ optou-se por realizar alguns experimentos precedentes aumentando a concentração de $\mathrm{Fe}^{0}$ presente (maior superfície de transferência). As condições experimentais realizadas e os resultados obtidos são mostrados na Tabela 6.

Tabela 6 - Testes para avaliação do aumento da concentração de $\mathrm{Fe}^{0}$ (POA foto-Fenton avançado).

\begin{tabular}{ccccc}
\hline & & & \multicolumn{2}{c}{ Redução } \\
\cline { 4 - 5 } Teste & $\mathbf{H}_{\mathbf{2}} \mathbf{O}_{\mathbf{2}}(\mathbf{m M})$ & $\mathbf{F e}^{\mathbf{0}}(\mathbf{m M})$ & $\mathbf{C O T}(\boldsymbol{\%})$ & $\mathbf{D Q O}(\boldsymbol{\%})$ \\
\hline & 530 & 17,9 & $73,4^{(1)}$ & $37,4^{(1)}$ \\
2 & 530 & 26,9 & 73,5 & 40,9 \\
3 & 530 & 35,8 & $\mathbf{7 4 , 1}$ & $\mathbf{4 1 , 0}$ \\
4 & 707 & 35,8 & 72,5 & 35,3 \\
\hline
\end{tabular}

(1) Valores médios dos resultados dos experimentos centrais.

Para redução de COT foi observado que o aumento das concentrações de $\mathrm{H}_{2} \mathrm{O}_{2}$ e $\mathrm{Fe}^{0}$ adicionados ao sistema não geraram melhores resultados, sendo assim, foi possível supor que quantidades ótimas devem estar em torno das condições experimentais do teste 2. A partir destes resultados foram propostos os níveis para o DCCR, sendo para o $\mathrm{H}_{2} \mathrm{O}_{2}(530-707 \mathrm{mM}) \mathrm{e}$ o $\mathrm{Fe}^{0}(17,9-35,8 \mathrm{mM})$ gerando a matriz experimental e, os resultados mostrados na Tabela 7 . 
Tabela 7 - Matriz experimental do DCCR e respectivas reduções de COT e DQO.

\begin{tabular}{|c|c|c|c|c|}
\hline \multirow[b]{2}{*}{ Exp. } & \multirow[b]{2}{*}{$\begin{array}{l}\mathrm{H}_{2} \mathrm{O}_{2} \\
(\mathbf{m M}) \\
\end{array}$} & \multirow[b]{2}{*}{$\mathrm{Fe}^{0}(\mathrm{mM})$} & \multicolumn{2}{|c|}{ Redução } \\
\hline & & & $\begin{array}{c}\text { COT } \\
(\%)\end{array}$ & $\begin{array}{c}\text { DQO } \\
(\%)\end{array}$ \\
\hline 1 & $557(-1)^{(1)}$ & $20,6(-1)$ & 73,3 & 40,4 \\
\hline 2 & $681(+1)$ & $20,6(-1)$ & 70,3 & 44,2 \\
\hline 3 & $557(-1)$ & $33,1(+1)$ & 72,9 & 39,6 \\
\hline 4 & $681(+1)$ & $33,1(+1)$ & 72,3 & 37,7 \\
\hline 5 & $530(-1,41)$ & $26,9(0)$ & 73,5 & 40,9 \\
\hline 6 & $707(+1,41)$ & $26,9(0)$ & 72,4 & 46,3 \\
\hline 7 & $619(0)$ & $17,9(-1,41)$ & 71,3 & 38,7 \\
\hline 8 & $619(0)$ & $35,8(+1,41)$ & 71,5 & 37,7 \\
\hline 9 & $619(0)$ & $26,9(0)$ & 74,3 & 43,7 \\
\hline 10 & $619(0)$ & $26,9(0)$ & 73,9 & 42,9 \\
\hline 11 & $619(0)$ & $26,9(0)$ & $\mathbf{7 4 , 0}$ & 42,0 \\
\hline
\end{tabular}

Analisando estes resultados é possível destacar que os experimentos realizados no ponto central mostraram os melhores resultados para redução de COT (valor médio de 74,1\%). Quanto aos valores de DQO, a maior redução alcançada foi de 46,3\% através do experimento 6. Pode-se observar que os experimentos com as menores concentrações de $\mathrm{Fe}^{0}$ foram aqueles que obtiveram as menores taxas de consumo de $\mathrm{H}_{2} \mathrm{O}_{2}$.

Após tratamento estatístico dos resultados foram geradas as superfícies de resposta e curvas de contorno para ambas as respostas (Figura 2). Finalizando, com o auxílio da função Desirability do software Minitab® e a partir dos modelos propostos foi determinada a condição ótima de reação para o uso do POA foto-Fenton avançado sobre o efluente em estudo. Os valores ótimos encontrados para as concentrações de $\mathrm{H}_{2} \mathrm{O}_{2}$ e $\mathrm{Fe}^{0}$ foram de $580 \mathrm{mM}$ e 26,6 mM, respectivamente. Foi realizada a validação experimental na condição ótima, obtendo reduções de 74,9\% em COT de e 41,0\% em DQO.

(a)

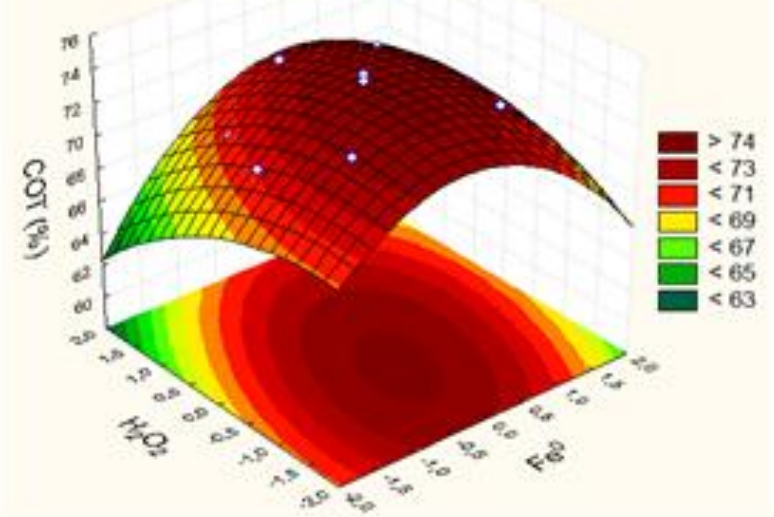

(b)

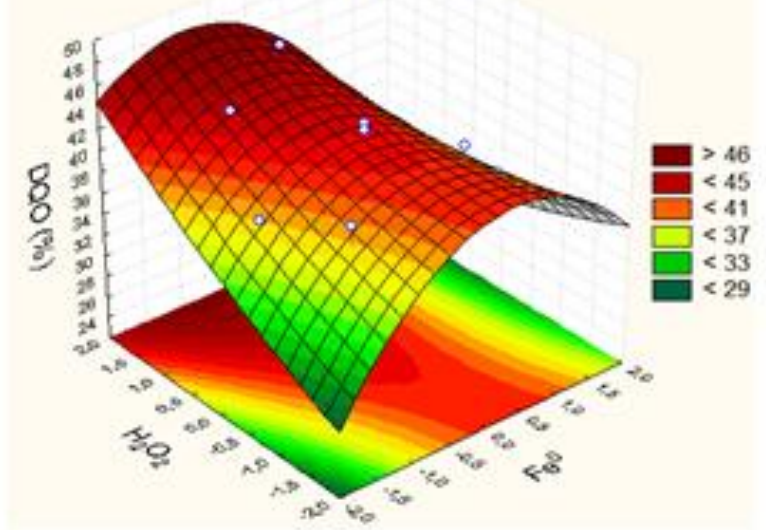

Figura 2 - Superfícies de resposta e curvas de contorno para as reduções de (a) COT e (b) DQO. 


\section{CONCLUSÃO}

Através dos resultados obtidos, pode-se concluir que o sistema foto-Fenton avançado propiciou reduções significativas de COT e DQO, mas ainda não totalmente satisfatórias quando considerado os níveis exigidos pela legislação ambiental, necessitando de ajustes quanto à forma de usar $\mathrm{o} \mathrm{Fe}^{0}$ no meio reacional de maneira mais eficiente. Deve ser considerada ainda a possibilidade de associação a outras técnicas de tratamento visando atingir níveis maiores de remoção de contaminantes.

O planejamento de experimentos mostrou-se como uma importante ferramenta estatística na obtenção da condição ótima considerando as variáveis de controle escolhidas. Através da análise estatística foram determinadas as concentrações de $\mathrm{H}_{2} \mathrm{O}_{2}$ e de $\mathrm{Fe}^{0}$ otimizadas para as máximas reduções de COT e DQO, com valores de redução de 74,9\% em COT, $41,0 \%$ em DQO e 30,3\% em DBO.

\section{REFERÊNCIAS}

BALANNEC, B.; VOURCH, M.; RABILLER-BAUDRY, M.; CHAUFER, M. Comparative study of different nanofiltration and reverse osmosis membranes for dairy effluent treatment by dead-end filtration. Separation and Purification Technology, v. 42, p. 195200, 2005.

BALDASSO, C.; BARROS, T.C.; TESSARO, I.C. Concentration and purification of whey proteins by ultrafiltration. Desalination, v. 278, p. 381-386, 2011.

BERGENDAHL, J.A.; THIES, T.P. Fenton's oxidation of MTBE with zero-valent iron. Water Research, v. 38, p. 327-324, 2004.

BREMNER, D.H.; BURGESS, A.E.; HOULLEMARE, D.; NAMKUNG, K.-C. Phenol degradation using hydroxyl radicals generated from zero-valent iron and hydrogen peroxide. Applied Catalysis B: Environmental, v. 63, p. 15-19, 2006

CUARTAS-URIBE, B.; ALCAINA-MIRANDA, M.I.; SORIANO-COSTA, E.; MENDOZAROCA, J.A.; IBORRA-CLAR, M.I.; LORA-GARCIA, J. A study of the separation of lactose from whey ultrafiltration permeate using nanofiltration. Desalination, v. 241, p. 244-255, 2009.

EPOLITO, W.J.; YANG, H.; BOTTOMLEY, L.A.; PAVLOSTATHIS, S.G. Kinetics of zerovalent iron reductive transformation of the anthraquinone dye Reactive Blue 4. Journal of Hazardous Materials, v. 160, p. 594-600, 2008.

GALVEZ, J.B.; RODRIGUEZ, S.M. Solar Detoxification. Almeria (Spain): United Nations Educational, Scientific and Cultural Organization, 2003.

JANCZUKOWICZ, W.; ZIELINSKI, M.; DEBOWSKI, M. Biodegrability evaluation of 
dairy effluents originated in selected sections of dairy production. Biosource Technology, v. 99, p. 4199-4205, 2008.

KUŠIĆ, H.; BOŽIĆ, A.L.; KOPRIVANAC, N. Fenton type processes for minimization of organic content in coloured wastewater: Part I: Processes optimization. Dyes and Pigments, v. 74, p. 380-387, 2007.

MAPA (Ministério da Agricultura, Pecuária e Abastecimento) - Sumário Executivo do Leite, Dezembro/2013. Disponível em: <http://www.agricultura.gov.br/politicaagricola/publicacoes/sumario-executivo> Acesso em: 04 abr 2014.

OPPENLÄNDER, T. Photochemical Purification of Water and Air. Germany: Wiley, 2003.

PEREIRA, C.D.; DIAZ, O.; COBOS, A. Valorization of by-products from ovine cheese manufacture: clarification by thermocalcic precipitation/microfiltration before ultrafiltration. International Dairy Journal, v. 12, p. 773-783, 2002.

PEREIRA, W.S.; FREIRE, R.S. Ferro zero: Uma nova abordagem para o tratamento de águas contaminadas com compostos orgânicos poluentes. Química Nova, v. 28, p. 130-136, 2005 .

PRAZERES, A.R.; CARVALHO, F; RIVAS, J. Cheese whey management: A review, Science of the Total Environment, v. 445-446, p. 385-396, 2012.

SOUZA, C.L.R. Degradação de corantes reativos e remediação de efluentes têxteis por processos avançados envolvendo ferro metálico. 2006. 82 f. Dissertação (Mestrado em Química Analítica) - Setor de Ciências Exatas, Departamento de Química, Universidade Federal do Paraná, Curitiba, 2006.

SOUZA, C.R.L.; PERALTA-ZAMORA, P. Degradação de corantes reativos pelo sistema ferro metálico/peróxido de hidrogênio. Química Nova, v. 28, p. 226-228, 2005.

ZHANG, H.; ZHANG, J.; ZHANG, C.; LIU, F.; ZHANG, D. Degradation of C.I. Acid Orange 7 by the advanced Fenton process in combination with ultrasonic irradiation. Ultrasonics Sonochemistry, v. 16, p. 325-330, 2009. 